\title{
Study on an 8-Wheel Suspension to Enhance the Hill-Climbing Performance for a Planetary Exploration Rover
}

\author{
We-Sub Eom ${ }^{\dagger}$ Joo-Hee Lee, Hyun-Cheol Gong and Gi-Hyuk Choi \\ Aerospace Convergence Technology Research Laboratory, Korea Aerospace Research Institute, Daejeon, 305-806, Korea
}

\begin{abstract}
Planetary exploration rovers are likely to make a trip on a winding and sloping road of irregular surfaces to the destination in order to accomplish scientific missions. One of the key technologies for rovers is a suspension for traveling and performing exploration missions; the suspension is an essential area of technology for a stable movement of a rover. In this study, an 8-wheel suspension is designed to enable efficient climbing of slopes on a passage to the destination. For the two front wheels among the eight wheels, the moment at the pivot connecting two wheels is derived when the distance between the wheels and the torque of wheels are same. A test experiment was performed to compare the magnitude of moment according to the change in tilt angle and the position of the pivot. Finally, a suspension design considering the position of the pivot was proposed to enhance the hill-climbing performance.
\end{abstract}

Keywords: planetary exploration rover, 8-wheel suspension, hill-climbing performance, moment at pivot, rocker-bogie suspension

\section{INTRODUCTION}

Planetary exploration rovers travel a road of unknown surfaces to the destination and perform scientific missions, such as, the investigation of geology and climate, the investigation of the existence of water and alien life forms (http://science.nasa.gov/platenary-science/missions). In order to perform these scientific missions successfully, the rover is developed to ensure the design targets: survival through the mission period and stability of movement to the destination (Eisen 1997, Siegwart et al. 2002, Chen et al. 2009). The typical suspensions applied to a conventional rover is a rocker-bogie suspension developed by NASA in 1960s $\sim 1970$ s (Eom et al. 2012a). This suspension was developed for a slowmoving system and 6-wheel application is enabled through this suspension structure. The rocker-bogie suspension undergoes a passive transformation in response to the shape of surfaces. Also it enables slope-climbing of 30 degree-tilt, and it is possible to maintain static equilibrium at the slope of 40 degree-tilt. The suspension of rocker-bogie type was applied to the first Mars Exploration Rover, Sojouner to prove its superior driving performance as shown in Fig. 1 through Fig. 3. It was subsequently applied to Spirit/Opportunity (http://marsrover.nasa.gov/home/index.html) and Curiosity (http://mars.jpl.nasa.gov/msl) (Bajracharya 2008, Eom et al. 2012a). Also at present, in addition to USA, developed nations in space technologies, such as, Europe, China, and Japan, etc., have had research projects on the suspension system of a rover lead by government funded research institutes and universities (Seeni et al. 2010, Ding et al. 2011, Trease et al. 2011).

Rovers have sensors to detect obstacles or dangers in forward or backward directions to get to the destination safely. The sensor collects the data of obstacles, such as, height, width, etc., in its way. The rover analyzes the obtained data to evaluate the possibility of stepping over the obstacle, and when it seems efficient in driving stability and power consumption to step over than to circumvent the obstacle, the rover goes on to overcome the obstacle. Therefore, in order to take account of the driving stability and power management at the same time, it is essential to have a suspension design to enable the efficient overcoming (c) This is an Open Access article distributed under the terms of the Creative Commons Attribution Non-Commercial License (http:// creativecommons.org/licenses/by-nc/3.0/) which permits unrestricted non-commercial use, distribution, and reproduction in any medium, provided the original work is properly cited.
Received Nov 3, 2014 Revised Dec 2, 2014 Accepted Dec 2, 2014 †Corresponding Author

E-mail: wse@kari.re.kr, ORCID: 0000-0002-6748-3620

Tel: +82-42-860-2255, Fax: +82-42-870-3980 


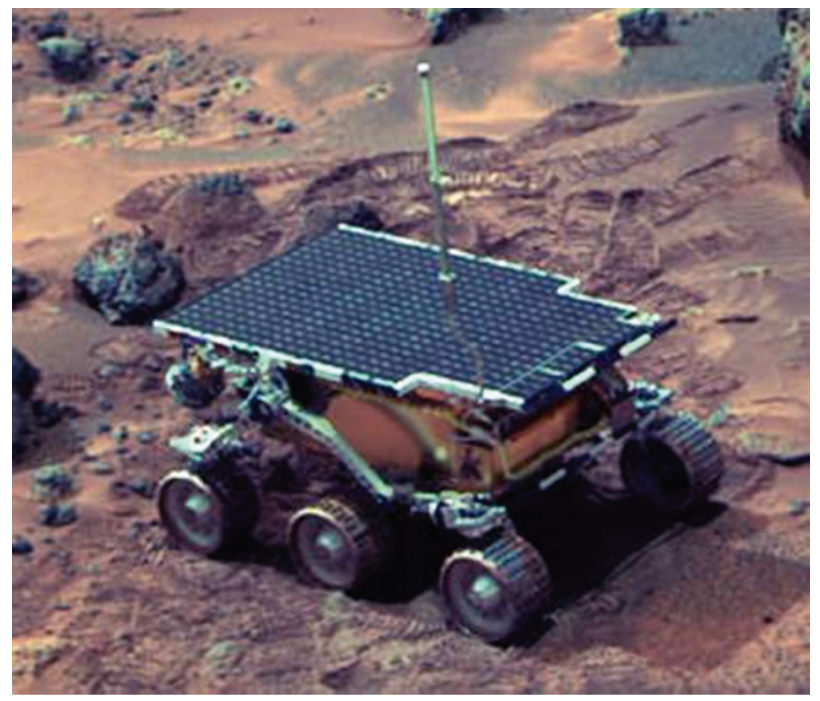

Fig. 1. Rover Sojourner.

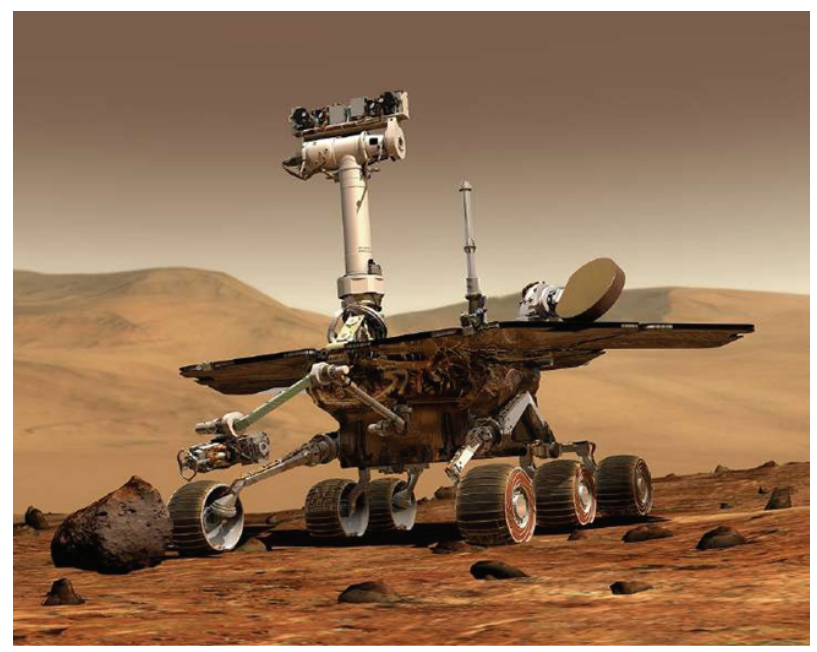

Fig. 2. Rover Spirit and Opportunity.

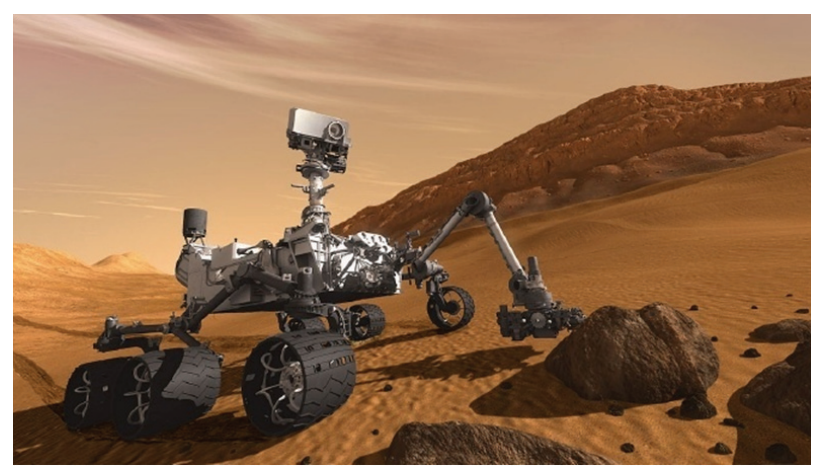

Fig. 3. Rover Curiosity. of obstacles (Kim et al. 2009, Eom et al. 2012b, Eom et al. 2014).

In this study, an 8-wheel suspension is designed to enable efficient climbing of slopes on a passage to the destination considering the pivot location to evaluate the climbing performance. In Section 2, the moment at the pivot connecting the two front wheels of a rover is derived, and in Section 3, in order to compare the hill-climbing performance, a test experiment is performed to compare the magnitude of moment according to the change in the tilt angle and the position of the pivot and the results are described. Finally, conclusion is summarized in the last section.

\section{DYNAMIC MODELING OF MOMENT ON PIVOT}

In this section, a conceptual outline is described and the necessary parameters are defined, for the proposed suspension. Also, the dynamic modeling on the above mentioned moment which arises during hill-climbing process is described. The outline of the 8 -wheel suspension for a planetary exploration rover is shown in Fig. 4 . The target of dynamic modeling is the center of rotation (the pivot; $C_{f}$ ) which connects the two wheels in the red rectangle as shown in Fig. 4.

For an 8-wheeled rover whose suspension system responds passively against tilted surfaces, the two front wheels' movement is divided into 4 stages as follows during the driving transition from flat surfaces to tilted surfaces. First, all wheels are on the flat surface and Wheel 1 touches the tilted surface to begin climbing the slope. Secondly, Wheel 1 is on the tilted surface and the Wheel 2 is on the flat surface. Thirdly, Wheel 1 is on the tilted surface and Wheel 2 begins climbing the slopes. Finally, both wheels are on the tilted surface to climb the slopes. Among the four stages, the first stage at which the moment start to arise at the pivot is described in this study. The Free Body Diagram (FBD) to derive the moment at the pivot is shown in Fig. 5.

Where, $\theta$ represents the tilted angle of the slopes, and $\mathrm{d}$ indicates the vertical distance from the pivot $\left(C_{f}\right)$ to the

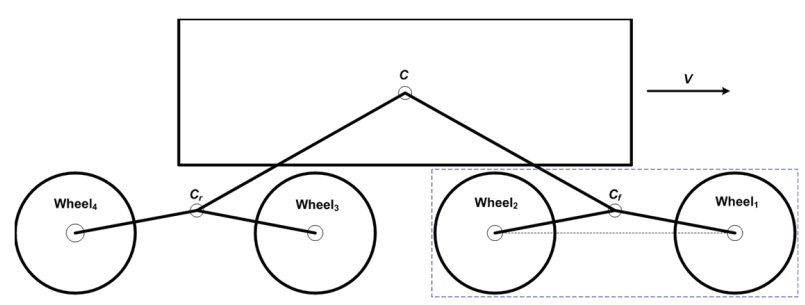

Fig. 4. Concept of Proposed Suspension for a Rover. 


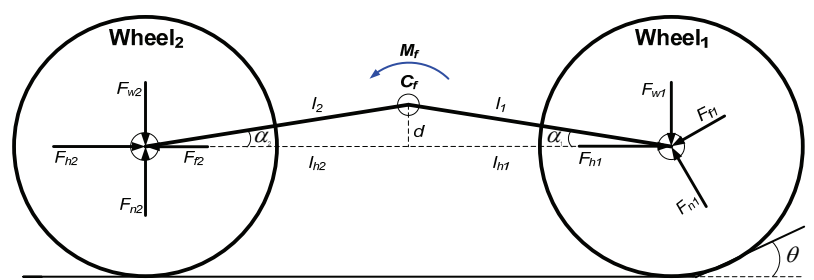

Fig. 5. FBD of the Front Suspension.

horizontal axis line connecting Wheel 1 and Wheel $2 ; l_{1}$ and $l_{2}$ are distances between the pivot $\left(C_{f}\right)$ and the centers of Wheel 1 and 2, respectively; $l_{h 1}$ and $l_{h 2}$ represent the horizontal distances between the pivot $\left(C_{f}\right)$ and the centers of Wheel 1 and 2, respectively; $\alpha_{1}$ and $\alpha_{2}$ are angles between $l_{1}$ and $l_{h 1}$, and between $l_{2}$ and $l_{h 2}$, respectively; $F_{h 1}$ and $F_{h 2}$ are driving forces acting on Wheel 1 and 2 by the motor torque; $F_{w 1}$ and $F_{w 2}$ are weights on Wheel 1 and $2 ; F_{n 1}$ and $F_{n 2}$ are normal force acting on Wheel land 2, respectively; $F_{f 1}$ and $F_{f 2}$ are friction force applied to Wheel 1 and 2, respectively; $M_{f}$ is the moment at the pivot $\left(C_{f}\right)$.

For ground moving robots, in order to identify the position of a robot, dynamics is derived considering the slipping effect between the wheel and the surface (Kozlowski \& Pazderski 2004), however, for planetary exploration rovers, since the sensor type and the methodology of position identification are different from those of ground moving robots, thus, this effect was deemed to be negligible. Moreover, due to the planetary surface environment which consists of irregular particles with various granularities, the friction coefficient term between the wheel and the surface is considered in the design of rover wheels, it is assumed to be same for the simplicity of equations.

From the FBD of Fig. 5, the derivation process of moment $\left(M_{f}\right)$ at the pivot $\left(C_{f}\right)$ is shown in Equation (1) through (5)

$$
\overrightarrow{F_{1}}=\overrightarrow{F_{h 1}}+\overrightarrow{F_{w 1}}+\overrightarrow{F_{n 1}}+\overrightarrow{F_{f 1}}
$$

where, $\overrightarrow{F_{h 1}}=F_{h 1} \vec{\imath}, \overrightarrow{F_{w 1}}=-F_{w 1} \vec{\jmath}, \overrightarrow{F_{n 1}}=F_{n 1}(-\sin \theta \vec{\imath}+$ $\cos \theta \vec{\jmath}), \overrightarrow{F_{f 1}}=F_{f 1}(-\cos \theta \vec{\imath}-\sin \theta \vec{\jmath})$.

$$
\overrightarrow{F_{2}}=\overrightarrow{F_{h 2}}+\overrightarrow{F_{w 2}}+\overrightarrow{F_{n 2}}+\overrightarrow{F_{f 2}}
$$

where, $\overrightarrow{F_{h 2}}=F_{h 2} \vec{\imath}, \overrightarrow{F_{w 2}}=-F_{w 2} \vec{\jmath}, \overrightarrow{F_{n 2}}=F_{n 2} \vec{\jmath}, \overrightarrow{F_{f 2}}=-F_{f 2} \vec{\imath}$.

$$
\overrightarrow{l_{1}}=l_{1}\left(\cos \alpha_{1} \vec{\imath}-\sin \alpha_{1} \vec{\jmath}\right)
$$

where, $l_{1}=\sqrt{l_{h 1}^{2}+d^{2}}, \quad \alpha_{1}=\operatorname{acos}\left(\frac{l_{h 1}}{l_{1}}\right)$

$$
\overrightarrow{l_{2}}=l_{2}\left(-\cos \alpha_{2} \vec{\imath}-\sin \alpha_{1} \vec{\jmath}\right)
$$

where, $l_{2}=\sqrt{l_{h 2}^{2}+d^{2}}, \alpha_{2}=\operatorname{acos}\left(\frac{l_{h 2}}{l_{2}}\right)$

$$
\overrightarrow{M_{f}}=\overrightarrow{l_{1}} \times \overrightarrow{F_{1}}+\overrightarrow{l_{2}} \times \overrightarrow{F_{2}}
$$

The larger the moment $\left(M_{f}\right)$ as derived in Equation (5) is, the less the friction force between Wheel 1 and the tilted surface. This indicates that the horizontal force acting on Wheel 1 on the slope decreases and the vertical force increases, thus, when climbing the tilted planetary surface of particles with low binding force such as a dry sand dune, the driving performance improves by not changing the shape of road surfaces.

\section{SIMULATION AND RESULTS}

Since the purpose of test experiment is to compare the hillclimbing performance according to the change in the position of pivot under the same driving environment, the effect of wheel slipping is neglected and same friction coefficients are assumed in this experiment. The tilted angle of slope is varied from $0^{\circ}$ to $40^{\circ}$ by $1^{\circ}$. While rovers are designed to be capable of climbing the slopes of maximum $30^{\circ}$ tilt in general (Eom et al. 2012a), the test condition was established considering the requirement to maintain static equilibrium at the slope of $40^{\circ}$. The properties of proposed suspension are summarized in Table 1. For simulations, the flowchart of the moment derivation process using force and position vectors defined in the FBD is shown in Fig. 6. Fig. 7 through Fig. 9 show the moment values $\left(M_{f}\right)$ according to the change in $d, l_{h 1}$, and $l_{h 2}$ as a function of tilt angle $(\theta)$ in the range of $0^{\circ} \sim 40^{\circ}$. Fig. 6 displays the behavior of the moment values when $\theta$ varies from $0^{\circ}$ to $40^{\circ}$ by the $1^{\circ}$ interval with fixed $l_{h 1}$ of $0.05 \mathrm{~m}$ and $l_{h 2}$ of $0.15 \mathrm{~m}$ for three kinds of $d: 0.0 \mathrm{~m}, 0.02 \mathrm{~m}$, and $0.04 \mathrm{~m}$. It was found that the larger the value of $d$ is, the bigger the value of the moment is up to the tilt angle of $28^{\circ}$, however, the moment value is larger for smaller $d$ with the tilt angle greater than $28^{\circ}$. The tilt angles giving 0 moment for each $\mathrm{d}$, 
Table 1. Properties about the proposed suspension.

\begin{tabular}{cc}
\hline Parameters & Value \\
\hline$l_{h 1}+l_{h 2}$ & $0.2 \mathrm{~m}$ \\
$F_{h 1}=F_{h 2}$ & $60 \mathrm{~N}$ \\
$F_{w 1}+F_{w 2}$ & $98 \mathrm{~N}$ \\
Friction coefficient $(\mu)$ & 0.7 \\
\hline
\end{tabular}

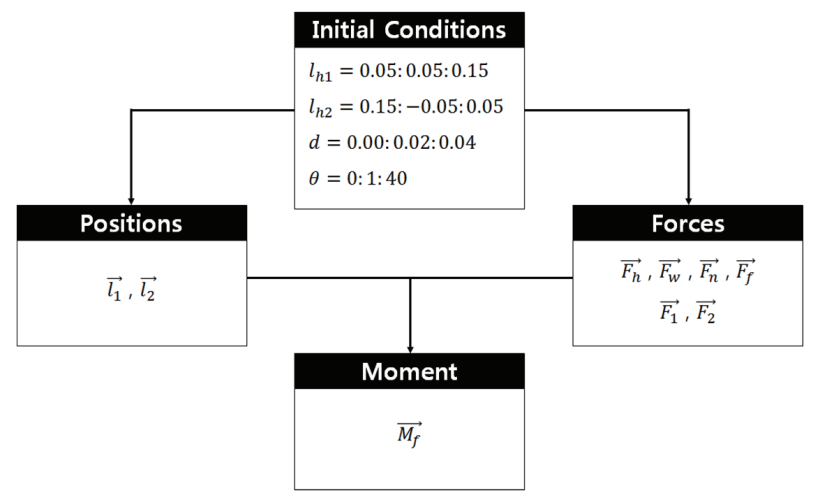

Fig. 6. FBD of the Front Suspension.

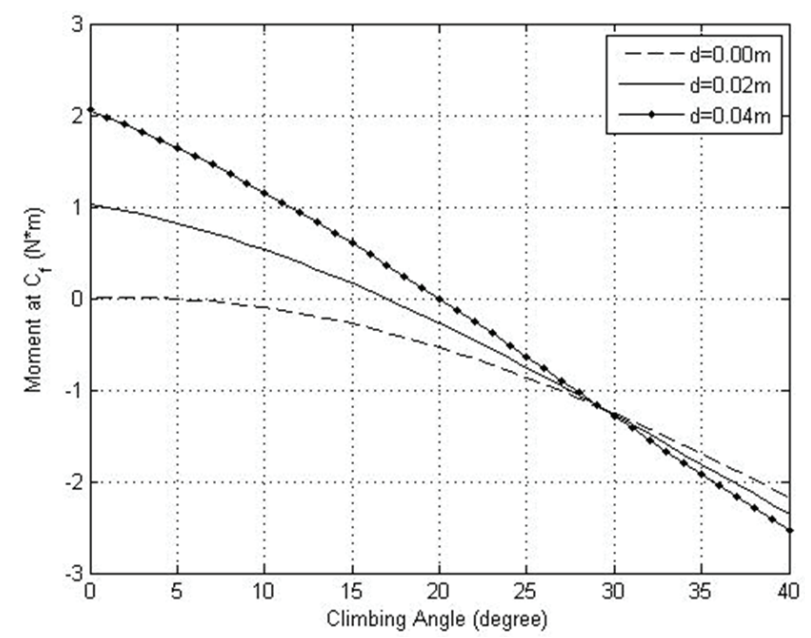

Fig. 7. Comparison for moments with $I_{h 1}=0.05 \mathrm{~m} \& I_{h 2}=0.15 \mathrm{~m}$.

turned out to be $0^{\circ}, 17^{\circ}$, and $25^{\circ}$, respectively. Thus, for the tilt angle greater than these, hill-climbing performance is degraded because the moment value becomes negative.

Fig. 8 shows the behavior of the moment values when $\theta$ varies from $0^{\circ}$ to $40^{\circ}$ by the $1^{\circ}$ interval with both $l_{h 1}$ and $l_{h 2}$ fixed to $0.15 \mathrm{~m}$ for three kinds of $d: 0.0 \mathrm{~m}, 0.02 \mathrm{~m}$, and $0.04 \mathrm{~m}$. The figure indicates that the larger the value of $d$ is, the bigger the value of the moment is up to the tilt angle of $35^{\circ}$, however, the moment value is larger for smaller $\mathrm{d}$ with the tilt angle greater than $38^{\circ}$. The tilt angles giving 0 moment for each $d$, turned out to be $16^{\circ}, 22^{\circ}$, and $24^{\circ}$, respectively. Thus, for the tilt angle greater than these, hill-climbing

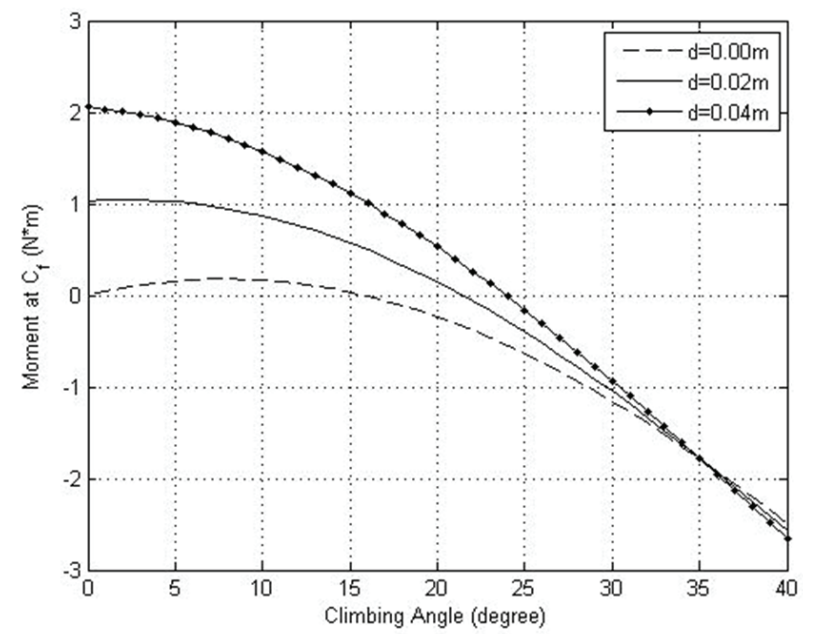

Fig. 8. Comparison for moments with $I_{h 1}=0.10 \mathrm{~m} \& I_{h 2}=0.10 \mathrm{~m}$.

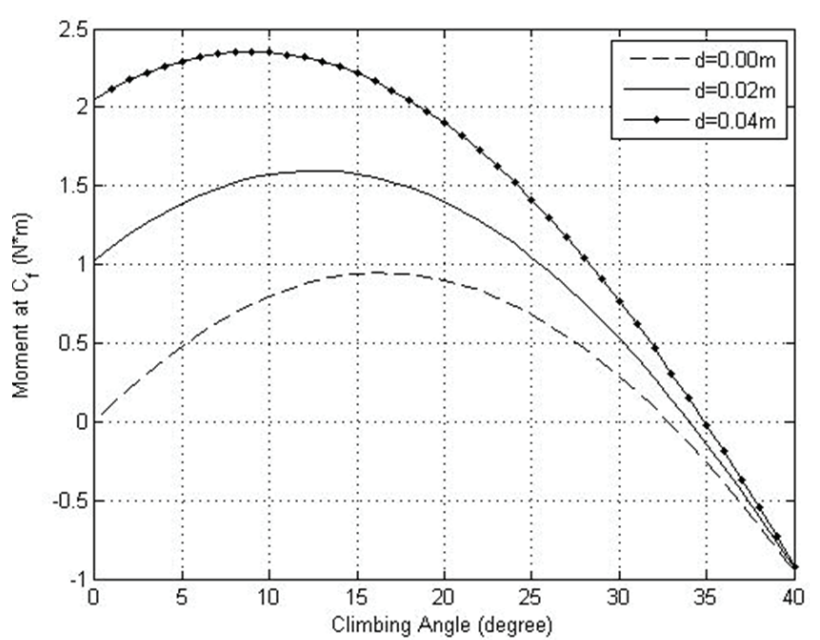

Fig. 9. Comparison for moments with $I_{h 1}=0.15 \mathrm{~m} \& I_{h 2}=0.05 \mathrm{~m}$.

performance is degraded because the moment value becomes negative.

Fig. 9 displays the behavior of the moment values when $\theta$ varies from $0^{\circ}$ to $40^{\circ}$ by the $1^{\circ}$ interval with fixed $l_{h 1}$ of $0.15 \mathrm{~m}$ and $l_{h 2}$ of $0.05 \mathrm{~m}$ for three kinds of $d: 0.0 \mathrm{~m}, 0.02 \mathrm{~m}$, and $0.04 \mathrm{~m}$. It was found that the larger the value of $d$ is, the bigger the value of the moment is up to the tilt angle of $40^{\circ}$ which is the upper end of the tilt angle range. The tilt angles giving 0 moment for each d, turned out to be $33^{\circ}, 34^{\circ}$, and $35^{\circ}$, respectively. Thus, for the tilt angle greater than these, hill-climbing performance is degraded because the moment value becomes negative.

\section{CONCLUSIONS}

In this study, for a planetary exploration rover which 
is required to overcome the obstacles in its way to the destination, a suspension design to improve the hillclimbing performance is proposed. The moment at the pivot which connects the two front wheels was derived for the proposed suspension system, and the change in the moment values were compared varying the vertical and horizon distance of the pivot according to the change in tilt angle.

According to the result of test experiment, as the pivot position is higher against the mid-point of horizontal line which connects the two front wheels and the pivot position is horizontally closer to the second wheel, hill-climbing performance is improved. However, it is not sufficient to analyze the complete hill-climbing performance by the analysis of hill-climbing at the inception of slope, dynamic modeling for the whole hill-climbing process and simulation will be performed to verify the excellence of current design for further study.

\section{REFERENCES}

Bajracharya M, Maimone M, Helmick D, Autonomy for Mars Rovers: Past, Present, and Future, Journal of the IEEE Computer Society, 41, 44-50 (2008).

Chen B, Wang R, Jia Y, Guo L, Yang L, Design of a High Performance Suspension for Lunar Rover Based on Evolution, Acta Astronautica, 64, 925-934 (2009).

Ding L, Gao H,Deng Z, Nagatani K, Yoshida, K, Experimental Study and Analysis on Driving Wheels' Performance for Planetary Exploration Rovers Moving in Deformable Soil, Journal of Terramechanics, 48(1), 27-45 (2011).

Eisen H, Buck W, Gillis-Smith R, Umland W, Mechanical Design of the Mars Pathfinder Mission, Proceedings of Seventh European Space Mechanisms and Tribology Symposium, ESA Headquarters, Noordwijk, the Netherlands, pp. 11-17 (1997).

Eom W-S, Kim Y-K, Lee J-H, Choi G-H, Sim E-S, Development Trend of Unmanned Planetary Exploration Rover by NASA, Current Industrial and Technology Trends in Aerospace, 10(2), 102-111 (2012a).

Eom W-S, Kim Y-K, Lee J-H, Choi G-H, Sim E-S, Study on a Suspension of a Planetary Exploration Rover to Improve Driving Performance During Overcoming Obstacles, Journal of Astronomy and Space Sciences, 29(4), 381387 (2012b).

Eom W-S, Lee J-H, Gong H-C, Choi G-H, Study of a Lightweight 8-Wheel Suspension to Improve the Hill-Climbing Ability for a Planetary Exploration Rover, The Korea Institute of Military Science and Technology 2014 Annual Conference,
1629-1630 (2014).

Kim Y-K, Kim H-D, Lee J-H, Sim E-S, Jeon S-W, Conceptual Design of Rover's Mobility System for Ground-Based Model, Journal of Astronomy and Space Sciences, 26, 677-692 (2009).

Kozlowski K, Pazderski D, Modeling and control of a 4-wheel skid-steering mobile robot, Int. J. Appl. Math. Comput. Sci., 14, 477-496 (2004).

Seeni A, Schafer B, Hirzinger G, Robot Mobility Systems for Planetary Surface Exploration - State-of-the-Art and Future Outlook: A Literature Survey, Aerospace Technologies Advancements, 189-208 (2010).

Siegwart R, Lamon P, Estier T, Lauria M, Innovative Design for Wheeled Locomotion in Rough Terrain, Robotics and Autonomous Systems 40(2), 151-162 (2002).

Trease B, Arvidson R, Lindemann R, Bennett K, Zhou F, et al., Dynamic Modeling and Soil Mechanics for Path Planning of the Mars Exploration Rovers, ASME 2011 International Design Engineering Technical Conferences and Computers and Information in Engineering Conference, 755-765, (2011). 